\title{
The crystal structure of girvasite, $\mathrm{NaCa}_{2} \mathrm{Mg}_{3}\left(\mathrm{PO}_{4}\right)_{3}\left(\mathrm{CO}_{3}\right)\left(\mathrm{H}_{2} \mathrm{O}\right)_{6}$, a complex phosphate carbonate hydrate based upon electroneutral heteropolyhedral layers
}

\author{
S.V. Krivovichev ${ }^{a, b, *}$, A.P. Chernyatieva ${ }^{a}$, S.N. Britvin ${ }^{a, b}$, V.N. Yakovenchuk ${ }^{b}$ \\ ${ }^{a}$ St. Petersburg State University, Department of Crystallography, Universitetskaya Nab. 7/9, St. Petersburg, 199034, Russia \\ ${ }^{\mathrm{b}}$ Nanomaterials Research Centre, Kola Science Centre, Russian Academy of Sciences, ul. Fersmana 14, Apatity, Murmansk Region, 184200, Russia
}

Received 18 June 2014; accepted 25 July 2014

Available online xx January 2015

\begin{abstract}
The crystal structure of girvasite, $\mathrm{NaCa}_{2} \mathrm{Mg}_{3}\left(\mathrm{PO}_{4}\right)_{3}\left(\mathrm{CO}_{3}\right)\left(\mathrm{H}_{2} \mathrm{O}\right)_{6}$, has been refined using X-ray diffraction data collected at $173 \mathrm{~K}$, which allowed to revise its crystal chemical formula. The mineral is monoclinic, $P 2{ }_{1} / c, a=6.4784(2), b=12.2313(3), c=21.3494(6) \AA$, $\beta=89.624(2)^{\circ}$, $V=1691.67(8) \AA^{3}$ (at $\left.173 \mathrm{~K}\right), Z=4, R_{1}=0.037$ for 6471 unique observed reflections. The crystal structure of girvasite contains three Mg, two $\mathrm{Ca}$ and one $\mathrm{Na}$ sites. The $\mathrm{Mg}$ atoms are octahedrally coordinated by $\mathrm{O}$ atoms and $\mathrm{H}_{2} \mathrm{O}$ molecules. The Ca sites are coordinated by eight anions each, whereas the $\mathrm{Na}$ site has a coordination number equal to seven. The crystal structure is based upon heteropolyhedral sheets formed by polymerization of $\mathrm{Mg}$ octahedra, $\mathrm{PO}_{4}$ tetrahedra and $\mathrm{CO}_{3}$ groups. The sheets consist of fundamental building units (FBBs) formed by two $\mathrm{Mg}$ octahedra that share edges to form dimers decorated by three $\mathrm{PO}_{4}$ tetrahedra and linked to a unit consisting of a $\mathrm{MgO}_{6}$ octahedron sharing an edge with a carbonate triangle. The FBBs polymerize to form chains running parallel to the $a$ axis. The chains are further polymerized to compose heteropolyhedral sheets stuffed by the $\mathrm{Ca}^{2+}$ and $\mathrm{Na}^{+}$cations, and $\mathrm{H}_{2} \mathrm{O}$ groups to form electroneutral layers parallel to (001). The adjacent layers connect to each other via a complex system of hydrogen bonds. The interesting feature of the structure is a bidentate coordination of $\mathrm{Mg} 2$ atom by a $\mathrm{CO}_{3}$ group. The $\mathrm{O} 14-\mathrm{Mg} 2-\mathrm{O} 16$ angle is shortened from $90^{\circ}$ (expected for a regular octahedron) to $60.46^{\circ}$, whereas the $\mathrm{O} 14-\mathrm{C}-\mathrm{O} 16$ angle is shortened from $120^{\circ}$ to $115.92^{\circ}$. Girvasite is the most structurally complex mineral among natural phosphate carbonates known to date. Its high structural complexity reflects its chemical complexity and high hydration state, which are the result of the specific geochemical and thermodynamic conditions of its formation (low-temperature and low-pressure interactions of phosphate-bearing solutions with primary dolomite carbonatites)..
\end{abstract}

(C) 2015, V.S. Sobolev IGM, Siberian Branch of the RAS. Published by Elsevier B.V. All rights reserved.

Keywords: girvasite; crystal structure; phosphate-carbonate; complexation; structural complexity; Kola Peninsula

\section{Introduction}

Structural and chemical diversity of natural carbonates continues to attract attention of mineralogists and petrologists with several new mineral species discovered and crystal structures determined within recent few years (Lazic et al., 2011; Bindi et al., 2011; Mills et al., 2012ab; Pekov et al., 2012; McDonald et al., 2012; Miyawaki et al., 2012; Biagioni et al., 2013; Kampf et al., 2014; Elliot et al., 2014, etc.). One of the source of variable rare and exotic carbonate minerals are carbonatites that have been subject for a large number of recent detailed experimental studies (Shatskiy et al., 2013abc, 2014).

\footnotetext{
* Corresponding author.

E-mail address: s.krivovichev@ spbu.ru (S.V. Krivovichev)
}

Girvasite, a rare hydrous $\mathrm{Na}-\mathrm{Ca}-\mathrm{Mg}$ phosphate carbonate, has been first described by Britvin et al. (1990) from hydrothermal veins in dolomite carbonatites of the Zhelezny (Iron) Mine, Kovdor massif, Kola Peninsula, Russia. The mineral was probably the result of reaction of primary dolomite with phosphate-bearing hydrothermal solutions. The Zhelezny mine is known as a type locality for several phosphate minerals, including rimkorolgite, $\mathrm{BaMg}_{5}\left(\mathrm{PO}_{4}\right)_{4}$. $\left(\mathrm{H}_{2} \mathrm{O}\right)_{8}$ (Britvin et al., 1995; Krivovichev et al., 2002), strontiowhitlockite, $\mathrm{Sr}_{9} \mathrm{Mg}\left(\mathrm{PO}_{3} \mathrm{OH}\right)\left(\mathrm{PO}_{4}\right)_{6}$ (Britvin et al., 1991), bakhchisaraitsevite, $\mathrm{Na}_{2} \mathrm{Mg}_{5}\left(\mathrm{PO}_{4}\right)_{4}\left(\mathrm{H}_{2} \mathrm{O}\right)_{7}$ (Liferovich et al., 2000; Yakubovich et al. 2000), cattiite, $\mathrm{Mg}_{3}\left(\mathrm{PO}_{4}\right)_{2}\left(\mathrm{H}_{2} \mathrm{O}\right)_{22}$ (Britvin et al. 2002; Chernyatieva et al., 2013), etc.

The crystal structure of girvasite was studied by Sokolova and Yegorov-Tismenko (1990), who assigned to the mineral the crystal chemical formula $\mathrm{NaCa}_{2} \mathrm{Mg}_{3}(\mathrm{OH})_{2} \mathrm{H}_{2}\left(\mathrm{PO}_{4}\right)_{3}\left(\mathrm{CO}_{3}\right)$. 
Table 1. Crystallographic data and refinement parameters for girvasite

\begin{tabular}{|c|c|}
\hline$a(\AA)$ & $6.4784(2)$ \\
\hline$b(\AA)$ & $12.2313(3)$ \\
\hline$c(\AA)$ & $21.3494(6)$ \\
\hline$\beta\left({ }^{\circ}\right)$ & $89.624(2)$ \\
\hline$V\left(\AA^{3}\right)$ & 1691.67(8) \\
\hline Space group & $P 2_{1} / c$ \\
\hline $\mathrm{F}_{000}$ & 1272 \\
\hline$\mu\left(\mathrm{cm}^{-1}\right)$ & 1.211 \\
\hline$D_{\text {calc }}\left(\mathrm{g} / \mathrm{cm}^{-1}\right)$ & 2.470 \\
\hline$Z$ & 4 \\
\hline Crystal size $\left(\mathrm{mm}^{3}\right)$ & $0.07 \times 0.09 \times 0.12$ \\
\hline Temperature (K) & 173 \\
\hline Radiation $\mathrm{MoK}_{\alpha}$ & 0.71073 \\
\hline $2 \Theta_{\max }$ & 75.56 \\
\hline Total Ref. & 55,166 \\
\hline Unique Ref. & 9000 \\
\hline Unique $\mid \mathrm{Fol} \leq 4 \sigma \mathrm{F}$ & 6471 \\
\hline$h_{\min }, h_{\max }$ & $-10,11$ \\
\hline$k_{\min }, k_{\max }$ & $-21,19$ \\
\hline$l_{\min }, l_{\max }$ & $-31,36$ \\
\hline$R_{\text {int }}$ & 0.0675 \\
\hline$R_{1}$ & 0.0373 \\
\hline$w R_{2}$ & 0.0839 \\
\hline$S$ & 1.022 \\
\hline$\rho_{\min }, \rho_{\max }\left(e \AA^{-3}\right)$ & $0.634,0.748$ \\
\hline
\end{tabular}

$\left(\mathrm{H}_{2} \mathrm{O}\right)_{4}$, which assumes the simultaneous presence in the structure of both protonated tetrahedral oxyanions and hydroxyl anions. Though such a combination is known for minerals (for instance, it has recently been described by Kampf et al. (2013) for joteite, $\mathrm{Ca}_{2} \mathrm{CuAl}\left[\mathrm{AsO}_{4}\right]\left[\mathrm{AsO}_{3}(\mathrm{OH})\right]_{2}(\mathrm{OH})_{2}$. $\left.5 \mathrm{H}_{2} \mathrm{O}\right)$, it is very unusual and requires additional verification. Since Sokolova and Yegorov-Tismenko (1990) were unable to determine positions of $\mathrm{H}$ atoms in girvasite, this problem remained unresolved and awaited special consideration. In this paper, we report on the crystal-structure determination of girvasite performed at $173 \mathrm{~K}$, which allowed us to identify hydrogen positions and to revise the crystal chemical formula of the mineral.

\section{Experimental}

The crystal of girvasite selected for data collection was mounted on a thin glass fiber for the X-ray diffraction analysis. More than a hemisphere of X-ray diffraction data with frame widths of $0.3^{\circ}$ in $\omega$, and $30 \mathrm{~s}$ spent counting for each frame were collected at $173 \mathrm{~K}$ using a Bruker four-circle Smart APEX DUO X-ray diffractometer operated at $50 \mathrm{kV}$ and $40 \mathrm{~mA}$ with $\mathrm{MoK}_{\alpha}$ radiation. The data were integrated and corrected for absorption using an empirical ellipsoidal model by means of the Bruker programs APEX and XPREP. The observed systematic absences were consistent with the space group $P 2_{1} / \mathrm{c}$ reported for girvasite by Sokolova and YegorovTismenko (1990). The structure was refined to $R_{1}=0.052$ using the atom coordinates for nonhydrogen atoms taken from Sokolova and Yegorov-Tismenko (1990). The inspection of difference Fourier electron-density map revealed the presence of twelve symmetrically independent $\mathrm{H}$ atoms that were incorporated into the structure refinement without any restraints. Structure refinement of the full structure model resulted in the crystallographic agreement index $R_{1}=0.038$ (Table 1). The SHELX program package was used for all structure calculations (Sheldrick, 2008). The final model included anisotropic displacement parameters for all nonhydrogen atoms atoms. The final atomic coordinates and anisotropic displacement parameters are given in Table 2 and selected interatomic distances are listed in Table 3.

\section{Results}

The structure of girvasite contains three $\mathrm{Mg}$, two $\mathrm{Ca}$ and one $\mathrm{Na}$ sites. The $\mathrm{Mg}$ atoms are octahedrally coordinated by $\mathrm{O}$ atoms and $\mathrm{H}_{2} \mathrm{O}$ molecules. The $\mathrm{Mg} 1$ site is coordinated by five $\mathrm{O}$ atoms and one $\mathrm{H}_{2} \mathrm{O}$ molecule, whereas the $\mathrm{Mg} 2$ and $\mathrm{Mg} 3$ sites are coordinated by four $\mathrm{O}$ atoms and two $\mathrm{H}_{2} \mathrm{O}$ molecules each. The $\mathrm{Ca}$ sites are coordinated by eight anions each, whereas the $\mathrm{Na}$ site has a coordination number equal to seven. The local coordination environments of the $\mathrm{Ca}$ and $\mathrm{Na}$ atoms are depicted in Figs. $1 a-c$.

The crystal structure of girvasite can be described as based upon heteropolyhedral sheets formed by polymerization of $\mathrm{Mg}$ octahedra, $\mathrm{PO}_{4}$ tetrahedra and $\mathrm{CO}_{3}$ triangular groups. The sheets consist of fundamental building units (FBBs) shown in Fig. 2. The FBB is formed by two $\mathrm{Mg}$ octahedra that share edges to form dimers decorated by three $\mathrm{PO}_{4}$ tetrahedra and linked to a unit consisting of a $\mathrm{MgO}_{6}$ octahedron sharing an edge with a triangular carbonate group. The FBBs polymerize to form chains running parallel to the $a$ axis (Fig. $3 a$ ). The chains are further polymerized to compose a heteropolyhedral sheet shown in Fig. $3 b$. The sheets are stuffed by the $\mathrm{Ca}^{2+}$ and $\mathrm{Na}^{+}$cations, and $\mathrm{H}_{2} \mathrm{O}$ groups to form electroneutral layers parallel to (001). The adjacent layers are connected to each other via a complex system of hydrogen bonds (Fig. 4).

\section{Discussion}

Carbonate groups in girvasite. The local coordination of carbonate groups in girvasite is shown in Fig. $1 d$. The most interesting and noteworthy feature of the structure is a bidentate (edge-sharing) coordination of $\mathrm{Mg} 2$ atom by a $\mathrm{CO}_{3}$ group. As a result, both $\mathrm{Mg}_{2} \mathrm{O}_{6}$ octahedron and $\mathrm{CO}_{3}$ group display considerable distortion. The $\mathrm{O} 14-\mathrm{Mg} 2-\mathrm{O} 16$ angle is shortened from $90^{\circ}$ (expected for a regular octahedron) to $60.46^{\circ}$, whereas the $\mathrm{O} 14-\mathrm{C}-\mathrm{O} 16$ angle is shortened from $120^{\circ}$ to $115.92^{\circ}$. In order to compensate for the angle shrinkage, 
Table 2. Atomic fractional coordinates and displacements parameters $\left(\AA^{2}\right)$ for the crystal structure of girvasite

\begin{tabular}{|c|c|c|c|c|}
\hline Atom & $x$ & $y$ & $z$ & $\mathrm{U}_{\mathrm{eq}}$ \\
\hline Ca1 & $0.43801(5)$ & $0.02010(2)$ & $0.249925(14)$ & $0.00498(6)$ \\
\hline $\mathrm{Ca} 2$ & $0.67971(5)$ & $0.25007(3)$ & $0.348619(14)$ & $0.00569(6)$ \\
\hline P1 & $0.43834(6)$ & $0.23332(3)$ & $0.719833(18)$ & $0.00503(7)$ \\
\hline $\mathrm{P} 2$ & $0.90536(6)$ & $0.07165(3)$ & $0.230884(18)$ & $0.00465(7)$ \\
\hline P3 & $0.75175(6)$ & $0.03410(3)$ & $0.594530(18)$ & $0.00535(7)$ \\
\hline $\mathrm{Mg} 1$ & $0.94562(8)$ & $0.15856(4)$ & $0.71836(2)$ & $0.00452(14)$ \\
\hline $\mathrm{Mg} 2$ & $0.62007(9)$ & $0.07221(5)$ & $0.11156(2)$ & $0.00610(15)$ \\
\hline $\mathrm{Mg} 3$ & $0.25003(8)$ & $0.02842(4)$ & $0.63224(2)$ & $0.00447(14)$ \\
\hline $\mathrm{Na}$ & $0.14773(11)$ & $0.20757(6)$ & $0.10527(3)$ & $0.00967(13)$ \\
\hline O1 & $0.27129(17)$ & $0.14992(9)$ & $0.69957(5)$ & $0.00614(19)$ \\
\hline $\mathrm{O} 2$ & $0.56724(18)$ & $0.00685(9)$ & $0.63779(5)$ & $0.0073(2)$ \\
\hline $\mathrm{O} 3$ & $0.91068(17)$ & $0.00191(9)$ & $0.75458(5)$ & $0.0065(2)$ \\
\hline $\mathrm{O} 4$ & $0.47074(18)$ & $0.18180(9)$ & $0.16814(5)$ & $0.0073(2)$ \\
\hline $\mathrm{O} 5$ & $0.37361(18)$ & $0.20668(9)$ & $0.28045(5)$ & $0.0072(2)$ \\
\hline O6 & $0.76394(18)$ & $0.00945(9)$ & $0.18514(5)$ & $0.0070(2)$ \\
\hline O7 & $0.97151(18)$ & $0.17966(9)$ & $0.20125(5)$ & $0.0068(2)$ \\
\hline O8 & $0.77604(18)$ & $0.08853(9)$ & $0.29220(5)$ & $0.0064(2)$ \\
\hline O9 & $0.63732(18)$ & $0.17091(9)$ & $0.73697(5)$ & $0.00642(19)$ \\
\hline $\mathrm{O} 10$ & $0.93679(17)$ & $0.08097(9)$ & $0.63212(5)$ & $0.0066(2)$ \\
\hline $\mathrm{O} 11$ & $0.17690(18)$ & $0.07033(9)$ & $0.43966(5)$ & $0.0074(2)$ \\
\hline $\mathrm{O} 12$ & $0.68505(18)$ & $0.12025(9)$ & $0.54579(5)$ & $0.0073(2)$ \\
\hline Ow13 & $0.02777(19)$ & $0.19752(10)$ & $0.81492(5)$ & $0.0077(2)$ \\
\hline $\mathrm{O} 14$ & $0.33790(19)$ & $0.05346(9)$ & $0.06098(5)$ & $0.0076(2)$ \\
\hline Ow15 & $0.79611(19)$ & $0.17783(10)$ & $0.05795(6)$ & $0.0092(2)$ \\
\hline O16 & $0.60557(18)$ & $0.05677(9)$ & $0.85916(5)$ & $0.0071(2)$ \\
\hline Ow17 & $0.2363(2)$ & $0.05055(10)$ & $0.94354(6)$ & $0.0082(2)$ \\
\hline Ow18 & $0.79694(19)$ & $0.11022(10)$ & $0.42313(5)$ & $0.0075(2)$ \\
\hline O19 & $0.82946(18)$ & $0.10907(10)$ & $0.93232(5)$ & $0.0088(2)$ \\
\hline Ow20 & $0.28478(19)$ & $0.13485(10)$ & $0.55629(6)$ & $0.0083(2)$ \\
\hline Ow21 & $0.4202(2)$ & $0.24607(11)$ & $0.42432(6)$ & $0.0112(2)$ \\
\hline $\mathrm{C}$ & $0.7053(2)$ & $0.03834(13)$ & $0.91051(7)$ & $0.0070(3)$ \\
\hline H131 & $0.131(4)$ & $0.236(2)$ & $0.8091(12)$ & $0.024(7)$ \\
\hline H132 & $0.079(4)$ & $0.141(2)$ & $0.8249(12)$ & $0.028(7)$ \\
\hline H151 & $0.752(5)$ & $0.249(2)$ & $0.0575(14)$ & $0.043(9)$ \\
\hline H152 & $0.797(5)$ & $0.157(2)$ & $0.0193(14)$ & $0.043(9)$ \\
\hline H171 & $0.263(5)$ & $0.054(2)$ & $0.9829(14)$ & $0.037(8)$ \\
\hline H172 & $0.113(4)$ & $0.062(2)$ & $0.9393(12)$ & $0.021(7)$ \\
\hline H181 & $0.921(5)$ & $0.105(2)$ & $0.4303(14)$ & $0.045(9)$ \\
\hline H182 & $0.740(5)$ & $0.116(3)$ & $0.4563(16)$ & $0.053(10)$ \\
\hline H201 & $0.403(4)$ & $0.135(2)$ & $0.5496(11)$ & $0.020(6)$ \\
\hline H202 & $0.233(4)$ & $0.109(2)$ & $0.5222(12)$ & $0.027(7)$ \\
\hline $\mathrm{H} 211$ & $0.357(4)$ & $0.300(2)$ & $0.4289(11)$ & $0.018(6)$ \\
\hline $\mathrm{H} 212$ & $0.328(4)$ & $0.187(2)$ & $0.4272(11)$ & $0.021(6)$ \\
\hline
\end{tabular}


Table 2 (continued)

\begin{tabular}{|c|c|c|c|c|c|c|}
\hline Atom & $\mathrm{U}_{11}$ & $\mathrm{U}_{12}$ & $\mathrm{U}_{13}$ & $\mathrm{U}_{23}$ & $\mathrm{U}_{13}$ & $\mathrm{U}_{12}$ \\
\hline $\mathrm{Ca} 1$ & $0.00456(12)$ & $0.00468(13)$ & $0.00571(12)$ & $0.00018(9)$ & $-0.00021(9)$ & $-0.00038(9)$ \\
\hline $\mathrm{Ca} 2$ & $0.00591(12)$ & $0.00536(13)$ & $0.00578(12)$ & $-0.00011(10)$ & $0.00005(9)$ & $0.00050(10)$ \\
\hline P1 & $0.00461(16)$ & $0.00477(17)$ & $0.00571(16)$ & $0.00005(13)$ & $-0.00014(12)$ & $-0.00019(13)$ \\
\hline $\mathrm{P} 2$ & $0.00452(16)$ & $0.00458(17)$ & $0.00486(16)$ & $-0.00020(12)$ & $-0.00021(12)$ & $0.00004(13)$ \\
\hline P3 & $0.00491(16)$ & $0.00592(17)$ & $0.00522(16)$ & $-0.00029(13)$ & $-0.00008(12)$ & $-0.00003(13)$ \\
\hline $\operatorname{Mg} 1$ & $0.0040(2)$ & $0.0042(2)$ & $0.0054(2)$ & $-0.00011(16)$ & $0.00015(16)$ & $0.00011(17)$ \\
\hline $\mathrm{Mg} 2$ & $0.0066(3)$ & $0.0064(3)$ & $0.0052(3)$ & $-0.00027(18)$ & $-0.00069(18)$ & $0.00064(19)$ \\
\hline $\mathrm{Mg} 3$ & $0.0043(2)$ & $0.0045(3)$ & $0.0046(2)$ & $-0.00036(17)$ & $-0.00028(16)$ & $0.00038(17)$ \\
\hline $\mathrm{Na}$ & $0.0101(3)$ & $0.0095(3)$ & $0.0095(3)$ & $-0.0002(2)$ & $0.0004(2)$ & $-0.0003(2)$ \\
\hline $\mathrm{O} 1$ & $0.0058(5)$ & $0.0052(5)$ & $0.0074(5)$ & $-0.0011(4)$ & $-0.0002(4)$ & $-0.0013(4)$ \\
\hline $\mathrm{O} 2$ & $0.0062(5)$ & $0.0096(5)$ & $0.0063(5)$ & $0.0006(4)$ & $0.0012(4)$ & $-0.0007(4)$ \\
\hline $\mathrm{O} 3$ & $0.0049(5)$ & $0.0059(5)$ & $0.0086(5)$ & $0.0006(4)$ & $-0.0011(4)$ & $0.0007(4)$ \\
\hline $\mathrm{O} 4$ & $0.0069(5)$ & $0.0065(5)$ & $0.0086(5)$ & $-0.0018(4)$ & $-0.0006(4)$ & $0.0006(4)$ \\
\hline O5 & $0.0073(5)$ & $0.0067(5)$ & $0.0076(5)$ & $0.0013(4)$ & $0.0006(4)$ & $0.0008(4)$ \\
\hline O6 & $0.0078(5)$ & $0.0066(5)$ & $0.0067(5)$ & $-0.0011(4)$ & $-0.0019(4)$ & $0.0001(4)$ \\
\hline O7 & $0.0074(5)$ & $0.0049(5)$ & $0.0081(5)$ & $0.0015(4)$ & $0.0010(4)$ & $-0.0005(4)$ \\
\hline O8 & $0.0074(5)$ & $0.0063(5)$ & $0.0056(5)$ & $0.0000(4)$ & $0.0004(4)$ & $0.0008(4)$ \\
\hline O9 & $0.0059(5)$ & $0.0060(5)$ & $0.0073(5)$ & $0.0005(4)$ & $-0.0006(4)$ & $0.0005(4)$ \\
\hline $\mathrm{O} 10$ & $0.0051(5)$ & $0.0079(5)$ & $0.0070(5)$ & $-0.0016(4)$ & $-0.0005(4)$ & $-0.0003(4)$ \\
\hline O11 & $0.0068(5)$ & $0.0074(5)$ & $0.0078(5)$ & $-0.0021(4)$ & $-0.0004(4)$ & $0.0007(4)$ \\
\hline $\mathrm{O} 12$ & $0.0067(5)$ & $0.0085(5)$ & $0.0066(5)$ & $0.0015(4)$ & $-0.0012(4)$ & $0.0007(4)$ \\
\hline Ow13 & $0.0070(5)$ & $0.0073(5)$ & $0.0087(5)$ & $0.0013(4)$ & $0.0007(4)$ & $-0.0005(4)$ \\
\hline $\mathrm{O} 14$ & $0.0105(5)$ & $0.0052(5)$ & $0.0071(5)$ & $0.0016(4)$ & $-0.0011(4)$ & $-0.0012(4)$ \\
\hline Ow15 & $0.0124(6)$ & $0.0074(5)$ & $0.0080(5)$ & $-0.0012(4)$ & $0.0003(4)$ & $0.0003(4)$ \\
\hline O16 & $0.0077(5)$ & $0.0069(5)$ & $0.0068(5)$ & $0.0003(4)$ & $-0.0017(4)$ & $-0.0002(4)$ \\
\hline Ow17 & $0.0089(5)$ & $0.0087(5)$ & $0.0070(5)$ & $-0.0005(4)$ & $-0.0005(4)$ & $0.0006(4)$ \\
\hline Ow18 & $0.0066(5)$ & $0.0094(5)$ & $0.0065(5)$ & $-0.0003(4)$ & $0.0000(4)$ & $0.0009(4)$ \\
\hline O19 & $0.0080(5)$ & $0.0092(5)$ & $0.0092(5)$ & $-0.0010(4)$ & $-0.0020(4)$ & $-0.0019(4)$ \\
\hline Ow20 & $0.0070(5)$ & $0.0111(5)$ & $0.0067(5)$ & $0.0003(4)$ & $-0.0009(4)$ & $-0.0008(4)$ \\
\hline Ow21 & $0.0105(5)$ & $0.0081(5)$ & $0.0149(6)$ & $-0.0001(4)$ & $0.0043(4)$ & $0.0007(4)$ \\
\hline $\mathrm{C}$ & $0.0071(6)$ & $0.0073(7)$ & $0.0066(6)$ & $-0.0001(5)$ & $0.0005(5)$ & $0.0011(5)$ \\
\hline
\end{tabular}

Table 3. Selected cation-anion bond lengths $(\AA)$ and parameters of the hydrogen bond system $(\AA$, deg) in the crystal structure of girvasite

\begin{tabular}{|c|c|c|c|c|c|}
\hline Ca1-O3 & $2.2776(12)$ & P2-O7 & $1.5250(12)$ & Mg3-O1 & $2.0727(12)$ \\
\hline Ca1-O9 & $2.4026(12)$ & $\mathrm{P} 2-\mathrm{O} 3$ & $1.5268(12)$ & $\mathrm{Mg} 3-\mathrm{O} 2$ & $2.0760(13)$ \\
\hline Ca1-O5 & $2.4090(12)$ & P2-O6 & $1.5439(12)$ & $\mathrm{Mg} 3-\mathrm{O} 20$ & $2.0903(13)$ \\
\hline $\mathrm{Ca} 1-\mathrm{O} 2$ & $2.4197(11)$ & $\mathrm{P} 2-\mathrm{O} 8$ & $1.5632(11)$ & $\mathrm{Mg} 3-\mathrm{O} 18$ & $2.0905(13)$ \\
\hline $\mathrm{Ca} 1-\mathrm{O} 8$ & $2.5173(12)$ & $<\mathrm{P} 2-\mathrm{O}>$ & 1.539 & $\mathrm{Mg} 3-\mathrm{O} 10$ & $2.1287(13)$ \\
\hline Ca1-O6 & $2.5199(12)$ & P3-O11 & $1.5407(12)$ & Mg3-O8 & $2.1619(12)$ \\
\hline Ca1-O16 & $2.5291(12)$ & $\mathrm{P} 3-\mathrm{O} 2$ & $1.5423(12)$ & $<\mathrm{Mg} 3-\mathrm{O}>$ & 2.102 \\
\hline $\mathrm{Ca} 1-\mathrm{O} 4$ & $2.6459(12)$ & P3-O12 & $1.5447(12)$ & $\mathrm{NaO} 20$ & $2.3632(14)$ \\
\hline$<\mathrm{Ca} 1-\mathrm{O}>$ & 2.465 & P3-O10 & $1.5564(12)$ & $\mathrm{NaO} 7$ & $2.3639(13)$ \\
\hline $\mathrm{Ca} 2-\mathrm{O} 21$ & $2.3251(13)$ & $<\mathrm{P} 3-\mathrm{O}>$ & 1.545 & $\mathrm{NaO} 14$ & $2.4392(13)$ \\
\hline
\end{tabular}


Table 3 (continued)

\begin{tabular}{|c|c|c|c|c|c|}
\hline Ca2-O8 & $2.3947(11)$ & $\mathrm{Mg} 1-\mathrm{O} 7$ & $2.0190(13)$ & $\mathrm{NaO} 4$ & $2.5133(13)$ \\
\hline $\mathrm{Ca} 2-\mathrm{O} 16$ & $2.4212(12)$ & Mg1-O9 & $2.0395(12)$ & $\mathrm{NaO} 15$ & $2.5243(15)$ \\
\hline $\mathrm{Ca} 2-\mathrm{O} 13$ & $2.4478(12)$ & $\mathrm{Mg} 1-\mathrm{O} 10$ & $2.0725(12)$ & $\mathrm{NaO} 1$ & $2.7845(13)$ \\
\hline $\mathrm{Ca} 2-\mathrm{O} 18$ & $2.4594(12)$ & $\mathrm{Mg} 1-\mathrm{O} 3$ & $2.0779(12)$ & $\mathrm{NaO} 10$ & $2.9792(13)$ \\
\hline $\mathrm{Ca} 2-\mathrm{O} 5$ & $2.5239(12)$ & $\mathrm{Mg} 1-\mathrm{O} 1$ & $2.1477(12)$ & $<\mathrm{Na}-\mathrm{O}>$ & 2.567 \\
\hline Ca2-O9 & $2.5884(11)$ & $\mathrm{Mg} 1-\mathrm{O} 13$ & $2.1852(13)$ & $\mathrm{C}-\mathrm{O} 14$ & $1.3066(19)$ \\
\hline $\mathrm{Ca} 2-\mathrm{O} 19$ & $2.6694(12)$ & $<\mathrm{Mg} 1-\mathrm{O}>$ & 2.089 & $\mathrm{C}-\mathrm{O} 16$ & $1.2958(19)$ \\
\hline$<\mathrm{Ca} 1-\mathrm{O}>$ & 2.478 & Mg2-O6 & $1.9862(12)$ & $\mathrm{C}-\mathrm{O} 19$ & $1.272(2)$ \\
\hline P1O4 & $1.5285(12)$ & Mg2-O4 & $2.0438(12)$ & $<\mathrm{C}-\mathrm{O}>$ & 1.291 \\
\hline P1O5 & $1.5433(12)$ & $\mathrm{Mg} 2-\mathrm{O} 15$ & $2.0646(13)$ & & \\
\hline P1O9 & $1.5442(12)$ & $\mathrm{Mg} 2-\mathrm{O} 17$ & $2.1190(13)$ & & \\
\hline P1O1 & $1.5508(12)$ & $\mathrm{Mg} 2-\mathrm{O} 14$ & $2.1416(13)$ & & \\
\hline \multirow[t]{2}{*}{$<\mathrm{P} 1-\mathrm{O}>$} & 1.541 & $\mathrm{Mg} 2-\mathrm{O} 16$ & $2.2370(13)$ & & \\
\hline & & $<\mathrm{Mg} 2-\mathrm{O}>$ & 2.098 & & \\
\hline \multicolumn{6}{|c|}{ Hydrogen bonding system } \\
\hline $\mathrm{D}-\mathrm{H}$ & $\mathrm{d}(\mathrm{D}-\mathrm{H}), \AA$ & $\mathrm{d}(\mathrm{H} \ldots \mathrm{A}), \AA$ & D-H...A & A & $\mathrm{d}(\mathrm{D} . . \mathrm{A}), \AA$ \\
\hline Ow13-H131 & 0.826 & 1.823 & 164.78 & $\mathrm{O} 5$ & 2.629 \\
\hline Ow13-H132 & 0.799 & 2.110 & 158.49 & O6 & 2.869 \\
\hline Ow15-H151 & 0.913 & 1.680 & 171.47 & $\mathrm{O} 12$ & 2.586 \\
\hline Ow15-H152 & 0.862 & 1.958 & 174.43 & O19 & 2.818 \\
\hline Ow17-H171 & 0.861 & 1.738 & 174.83 & $\mathrm{O} 14$ & 2.596 \\
\hline Ow17-H172 & 0.814 & 1.934 & 172.08 & O19 & 2.743 \\
\hline Ow18-H181 & 0.824 & 1.721 & 169.67 & O11 & 2.536 \\
\hline Ow18-H182 & 0.798 & 1.943 & 162.67 & $\mathrm{O} 12$ & 2.715 \\
\hline Ow20-H201 & 0.780 & 1.835 & 170.29 & $\mathrm{O} 12$ & 2.607 \\
\hline Ow20-H202 & 0.865 & 1.862 & 165.57 & O11 & 2.708 \\
\hline Ow21-H211 & 0.784 & 2.010 & 171.13 & O17 & 2.787 \\
\hline Ow21-H212 & 0.937 & 1.752 & 172.78 & O11 & 2.684 \\
\hline
\end{tabular}

Note. D, donor; A, acceptor.

Table 4. Phosphate-carbonate minerals with known crystal structures and their structural complexity parameters

\begin{tabular}{|c|c|c|c|c|c|}
\hline Mineral name & Chemical formula & $v$ [atoms] & $I_{G}$ [bits/at.] & $I_{G, t o t a l}[$ bits/u.c.] & Reference \\
\hline Abenakiite-(Ce) & $\mathrm{Na}_{26} \mathrm{Ce}_{6}\left(\mathrm{SO}_{2}\right)\left(\mathrm{SiO}_{3}\right)_{6}\left(\mathrm{PO}_{4}\right)_{6}\left(\mathrm{CO}_{3}\right)_{6}$ & 117 & 4.335 & 507.147 & (McDonald et al., 1994) \\
\hline Bonshtedtite & $\mathrm{Na}_{3} \mathrm{Fe}\left(\mathrm{PO}_{4}\right)\left(\mathrm{CO}_{3}\right)$ & 26 & 3.393 & 88.211 & (Krivovichev et al., 2013) \\
\hline Crawfordite & $\mathrm{Na}_{3} \mathrm{Sr}\left(\mathrm{PO}_{4}\right)\left(\mathrm{CO}_{3}\right)$ & 26 & 3.700 & 96.211 & (Sokolova and Khomyakov, 1992) \\
\hline Daqingshanite-(Ce) & $\mathrm{Sr}_{3} \mathrm{Ce}\left(\mathrm{PO}_{4}\right)\left(\mathrm{CO}_{3}\right)_{3}$ & 21 & 2.748 & 57.709 & (Hughes and Ni, 1994) \\
\hline Devitoite & $\left(\mathrm{Ba}_{6}\left(\mathrm{PO}_{4}\right)_{1.48} \mathrm{O}_{2.08}\left(\mathrm{CO}_{3}\right)\right)\left(\mathrm{Fe}_{9}(\mathrm{OH})_{4} \mathrm{O} 2\left(\mathrm{SiO}_{3}\right)_{8}\right)$ & 64 & 5.031 & 322.000 & (Kampf et al., 2010) \\
\hline Girvasite & $\mathrm{NaCa}_{2} \mathrm{Mg}_{3}\left(\mathrm{PO}_{4}\right)_{3}\left(\mathrm{CO}_{3}\right)\left(\mathrm{H}_{2} \mathrm{O}\right)_{6}$ & 172 & 5.426 & 933.318 & (This work) \\
\hline Heneuite & $\mathrm{CaMg}_{5}\left(\mathrm{CO}_{3}\right)\left(\mathrm{PO}_{4}\right)_{3}(\mathrm{OH})$ & 52 & 4.700 & 244.423 & (Romming and Raade, 1986) \\
\hline Peatite-(Y) & $\mathrm{Li}_{4} \mathrm{Na}_{12} \mathrm{Y}_{12}\left(\mathrm{PO}_{4}\right)_{12}\left(\mathrm{CO}_{3}\right)_{4}(\mathrm{~F}, \mathrm{OH})_{8}$ & 112 & 4.914 & 550.424 & (McDonald et al., 2013) \\
\hline Ramikite-(Y) & $\mathrm{Li}_{4} \mathrm{Na}_{12} \mathrm{Y}_{6} \mathrm{Zr}_{6}\left(\mathrm{PO}_{4}\right)_{12}\left(\mathrm{CO}_{3}\right)_{4} \mathrm{O}_{4}(\mathrm{OH}, \mathrm{F})_{4}$ & 112 & 6.807 & 762.424 & (McDonald et al., 2013) \\
\hline Sidorenkite & $\mathrm{Na}_{3} \mathrm{Mn}\left(\mathrm{PO}_{4}\right)\left(\mathrm{CO}_{3}\right)$ & 26 & 3.393 & 88.211 & (Kurova et al., 1980) \\
\hline Skorpionite & $\mathrm{Ca}_{3} \mathrm{Zn}_{2}\left(\mathrm{PO}_{4}\right)_{2}\left(\mathrm{CO}_{3}\right)(\mathrm{OH})_{2}\left(\mathrm{H}_{2} \mathrm{O}\right)$ & 50 & 3.844 & 192.193 & (Krause et al., 2008) \\
\hline Voggite & $\mathrm{Na}_{2} \mathrm{Zr}\left(\mathrm{PO}_{4}\right)\left(\mathrm{CO}_{3}\right)(\mathrm{OH})\left(\mathrm{H}_{2} \mathrm{O}\right)_{2}$ & 30 & 3.640 & 109.207 & (Szymanski and Roberts, 1990) \\
\hline
\end{tabular}

Note. $v$, Number of atoms per reduced unit cell; $I_{\mathrm{G}}$, structural information amount per atom; $I_{\mathrm{G}, \text { total }}$, structural information amount per unit cell. 

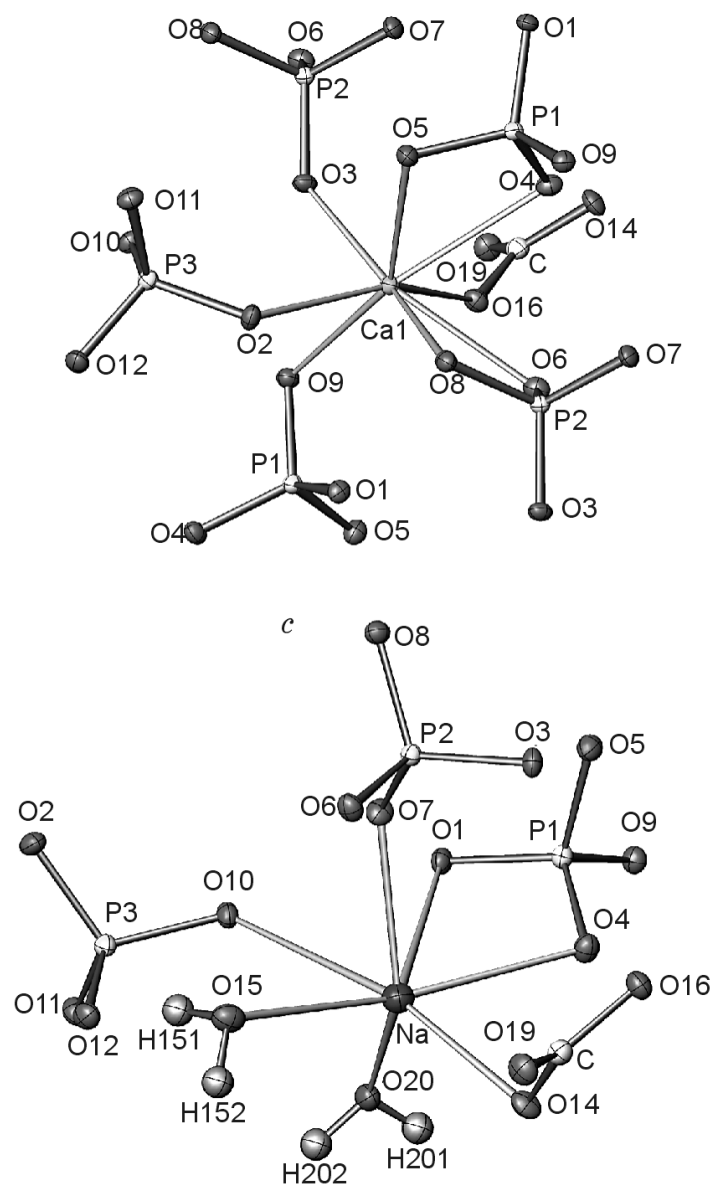

b

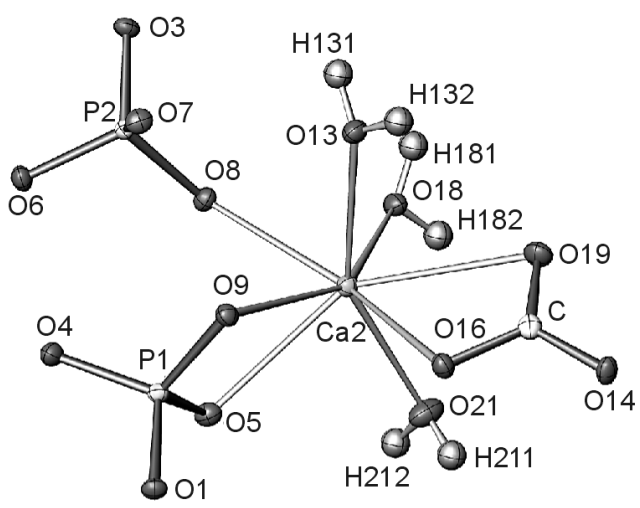

$d$

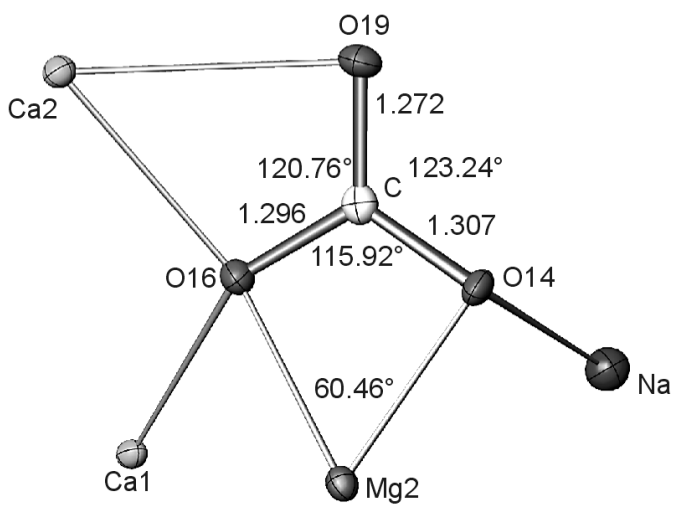

Fig. 1. Coordination environments of the $\mathrm{Ca}$ and $\mathrm{Na}$ sites $(a-c)$ and $\mathrm{CO}_{3}$ groups $(d)$ in the crystal structure of girvasite (atom displacement ellipsoids are drawn at $75 \%$ probability level).
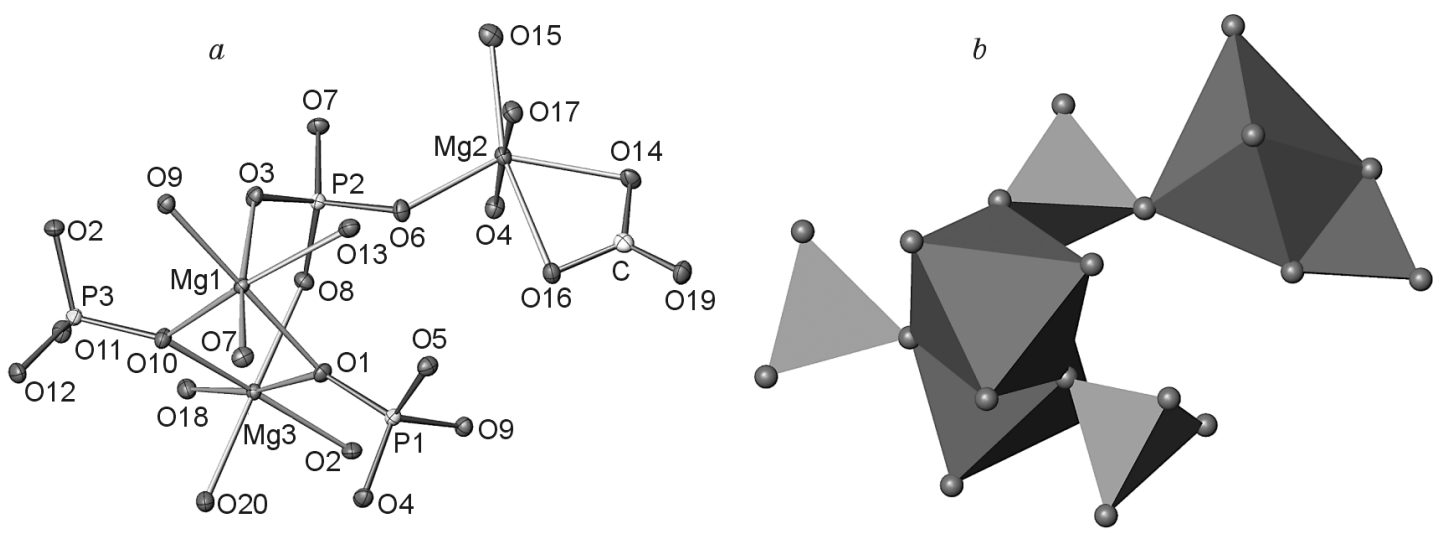

Fig. 2. Fundamental building block in the crystal structure of girvasite shown in ellipsoid $(a)$ and polyhedral $(b)$ representations (atom displacement ellipsoids are drawn at $75 \%$ probability level).

the $\mathrm{C}-\mathrm{O} 14$ and $\mathrm{C}-\mathrm{O} 16$ bonds are elongated compared to the $\mathrm{C}-\mathrm{O} 19$ bond (Fig. $1 d$ ). The bidentate mode of coordination of an octahedral di- or trivalent cation by carbonate is rare in minerals, but has been observed in the crystal structures of the bradleyite-group minerals (Kurova et al., 1980; Sokolova and Khomyakov, 1992; Krivovichev et al. 2013). Bidentate coordination is also common for natural uranyl carbonates (Krivovichev and Plásil, 2013), where $\mathrm{UO}_{2}^{2+}$ linear cations are bidentately coordinated by $\mathrm{CO}_{3}$ groups to form stable uranyl carbonate clusters that are known to persist in aqueous solutions and to serve as transport agents of uranium in natural and technological environments (Clark et al., 1995). It may 

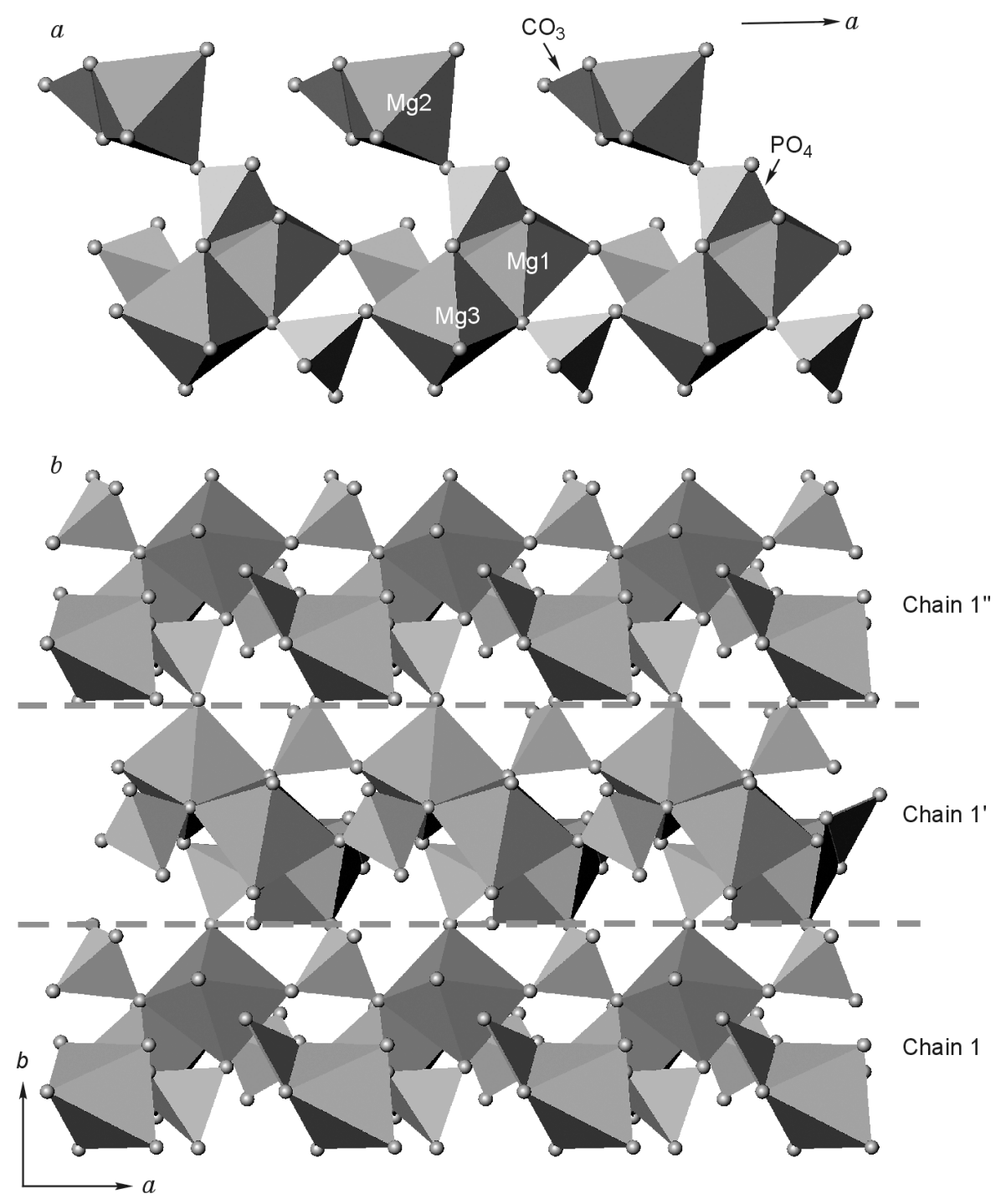

Fig. 3. One-dimensional polymerization of FBBs along the $a$ axis results in the formation of complex chains $(a)$, which are further linked along the $b$ axis to form two-dimensional sheets $(b)$ (dashed line shows the borders of the chains within the sheet).

be suggested that the bidentate complexation of $\mathrm{Mg}^{2+}$ cations by $\mathrm{CO}_{3}$ groups may occur in aqueous solutions under particular conditions such as those that led to the crystallization of girvasite.

Crystal chemical formula of girvasite. As it was mentioned above, the initial formula of girvasite proposed by Sokolova and Yegorov-Tismenko (1990) on the basis of crystal-structure determination is $\mathrm{NaCa}_{2} \mathrm{Mg}_{3}(\mathrm{OH})_{2} \mathrm{H}_{2}\left(\mathrm{PO}_{4}\right)_{3}\left(\mathrm{CO}_{3}\right)\left(\mathrm{H}_{2} \mathrm{O}\right)_{4}$. However, determination of all $\mathrm{H}$ sites in this work revealed no hydroxyl groups or protonated $\mathrm{PO}_{4}^{3-}$ anions. Instead, all $\mathrm{O}$ atoms not bonded to $\mathrm{P}^{5+}$ or $\mathrm{C}^{4+}$ cations form two strong hydrogen bonds each, thus belonging to $\mathrm{H}_{2} \mathrm{O}$ groups. This observation allows us to revise the crystal chemical formula of girvasite as $\mathrm{NaCa}_{2} \mathrm{Mg}_{3}\left(\mathrm{PO}_{4}\right)_{3}\left(\mathrm{CO}_{3}\right)\left(\mathrm{H}_{2} \mathrm{O}\right)_{6}$. This formula excludes simultaneous occurrence of the basic $(\mathrm{OH})^{-}$and acid $\left(\mathrm{PO}_{3} \mathrm{OH}\right)^{2-}$ anions originally postulated for girvasite by Sokolova and Yegorov-Tismenko (1990).

Structural complexity of girvasite and related minerals. The complexity of the atomic arrangement in girvasite can be quantitatively evaluated as an information amount in bits per unit cell $\left(I_{G, \text { total }}\right)$ using the formula (Krivovichev, 2013, 2014):

$I_{G, \text { total }}=-v I_{G}=-v \sum_{i=1}^{k} p_{i} \log _{2} p_{i}$ (bits/u.c.),

where $k$ is the number of different crystallographic orbits, $I_{G}$ is the information amount per atom, and $p_{i}$ is the random choice probability for an atom from the $i$ th crystallographic orbit, that is:

$p_{i}=m_{i} / v$,

where $m_{i}$ is a multiplicity of a crystallographic orbit relative to the reduced unit cell, and $v$ is the number of atoms in the reduced unit cell.

The $I_{G \text {,total }}$ value for girvasite is 933.318 bits per unit cell, which places it among the class of complex minerals (5001000 bits/u.c.). This relatively high level of complexity of the crystal structure of girvasite is comparable to that observed 


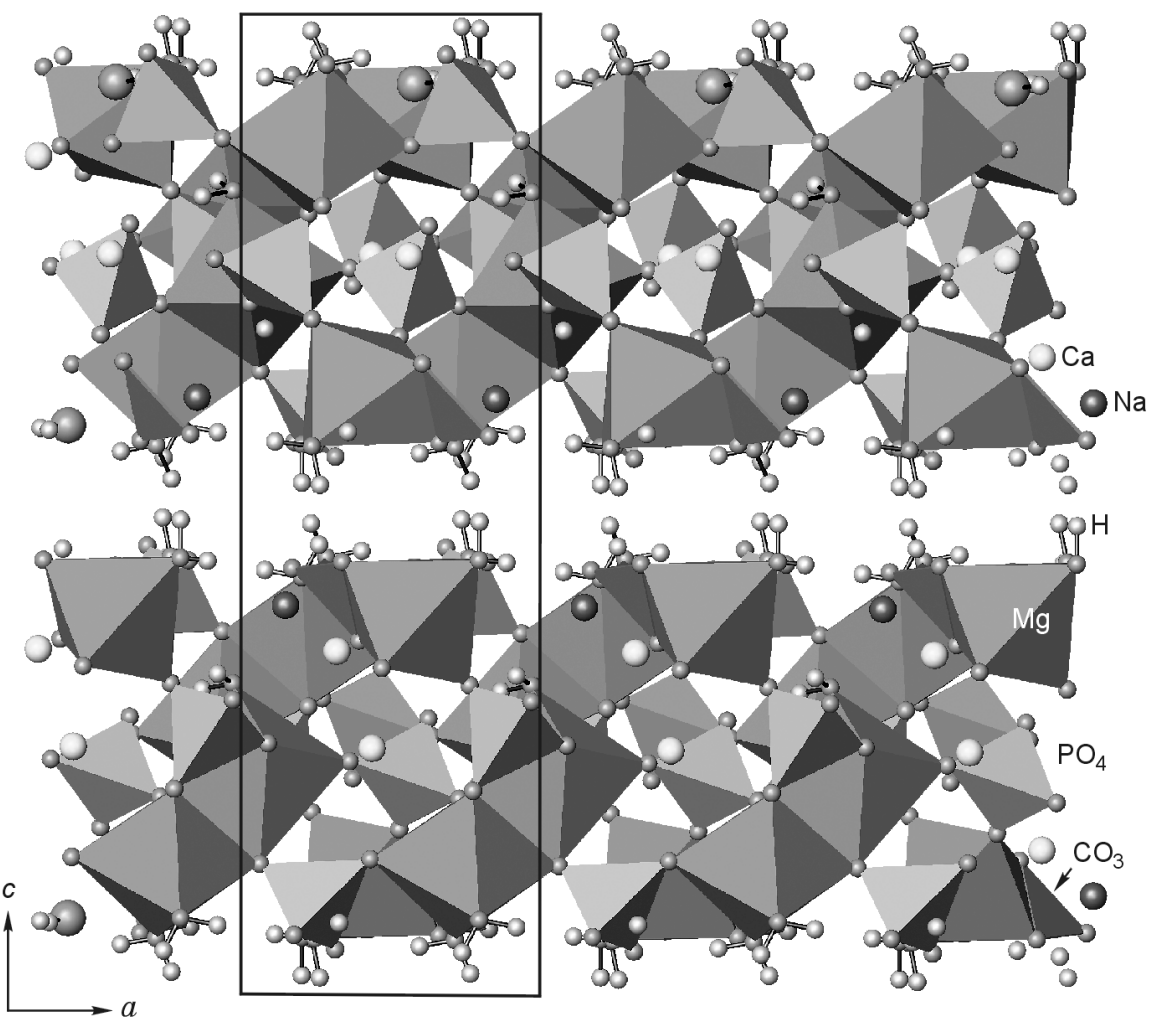

Fig. 4. The crystal structure of girvasite projected parallel to the $b$ axis.

for other rare Mg-bearing phosphate minerals first described from the Zhelezny mine of the Kovdor massif: rimkorolgite, $\mathrm{BaMg}_{5}\left(\mathrm{PO}_{4}\right)_{4}\left(\mathrm{H}_{2} \mathrm{O}\right)_{8}$ (691.895 bits/u.c.), bakhchisaraitsevite, $\mathrm{Na}_{2} \mathrm{Mg}_{5}\left(\mathrm{PO}_{4}\right)_{4}\left(\mathrm{H}_{2} \mathrm{O}\right)_{7} \quad(1128.771$ bits/u.c. $)$, cattiite, $\mathrm{Mg}_{3}\left(\mathrm{PO}_{4}\right)_{2}\left(\mathrm{H}_{2} \mathrm{O}\right)_{22}$ (419.999 bits/u.c.), and strontiowhitlockite, $\mathrm{Sr}_{9} \mathrm{Mg}\left(\mathrm{PO}_{3} \mathrm{OH}\right)\left(\mathrm{PO}_{4}\right)_{6}$ (375.031 bits/u.c.). The high structural complexity of the minerals clearly reflects their chemical complexity and high hydration states (except for strontiowhitlockite), which are the results of the specific geochemical and thermodynamic conditions (low-temperature and low-pressure interactions of phosphate-bearing solutions with primary dolomite carbonatites). It is of interest that girvasite is the most structurally complex mineral among natural phosphate carbonates known to date (see Table 4 for a list of phosphate-carbonate minerals and their structural complexity parameters).

Acknowledgements. This work was supported for SVK, APC and SNB by the Russian Foundation for Basic Research (grant \# 14-05-00910) and internal grant of St. Petersburg State University (\#3.38.136.2014). X-ray diffraction measurements have been performed at the SPbSU X-ray Diffraction Resource Centre.

\section{References}

Biagioni, C., Bonaccorsi, E., Camara, F., Cadoni, M., Ciriotti, M.E., Bersani, D., Kolitsch, U., 2013. Lusernaite-(Y), $\mathrm{Y}_{4} \mathrm{Al}\left(\mathrm{CO}_{3}\right)_{2}(\mathrm{OH}, \mathrm{F})_{11}$. $6 \mathrm{H}_{2} \mathrm{O}$; a new mineral species from Luserna Valley, Piedmont, Italy; description and crystal structure. Am. Mineral. 98, 1322-1329.
Bindi, L., Nestola, F., Kolitsch, U., Guastoni, A., Zorzi, F., 2011. Fassinaite, $\mathrm{Pb}_{2}^{2+}\left(\mathrm{S}_{2} \mathrm{O}_{3}\right)\left(\mathrm{CO}_{3}\right)$, the first mineral with coexisting thiosulphate and carbonate groups: description and crystal structure. Mineral. Mag. 75, 2721-2732.

Britvin, S.N., Pakhomovskii, Y.A., Bogdanova, A.N., Sokolova, E.V., 1990. Girvasite, a new carbonate-phosphate of sodium, calcium and magnesium. Mineralogicheskii Zh. 12, 79-83.

Britvin, S.N., Pakhomovskii, Y.A., Bogdanova, A.N., Skiba, V.I., 1991. Strontiowhitlockite, $\mathrm{Sr}_{9} \mathrm{Mg}\left(\mathrm{PO}_{3} \mathrm{OH}\right)\left(\mathrm{PO}_{4}\right)_{6}$, a new mineral species from the Kovdor deposit, Kola-Peninsula, USSR. Can. Mineral. 29, 87-93.

Britvin, S.N., Pakhomovskii, Y.A., Bogdanova, A.N., Khomyakov, A.P., Krasnova, N.I., 1995. Rimkorolgite, $(\mathrm{Mg}, \mathrm{Mn})_{5}(\mathrm{Ba}, \mathrm{Sr}, \mathrm{Ca})\left(\mathrm{PO}_{4}\right)_{4} \cdot 8 \mathrm{H}_{2} \mathrm{O}$, a new mineral. Zap. Vses. Mineral. Obshch. 124, 90-95

Britvin, S.N., Ferraris, G., Ivaldi, G., Bogdanova, A.N., Chukanov, N.V., 2002. Cattiite, $\mathrm{Mg}_{3}\left(\mathrm{PO}_{4}\right)_{2} \cdot 22 \mathrm{H}_{2} \mathrm{O}$, a new mineral from Zhelezny mine (Kovdor Massif, Kola Peninsula, Russia). N. Jb. Mineral. Mh. 2002, $160-168$.

Chernyatieva, A.P., Krivovichev, S.V., Britvin, S.N., 2013. The crystal structure of cattiite, $\mathrm{Mg}_{3}\left(\mathrm{PO}_{4}\right)_{2}\left(\mathrm{H}_{2} \mathrm{O}\right)_{22}$. Zap. Ross. Mineral. Obshch. 142 (2), 120-128 [Geol. Ore Deposits, 2014, in press].

Clark, D.L., Hobart, D.E., Neu, M.P., 1995. Actinide carbonate complexes and their importance in actinide environmental chemistry. Chem. Rev. 95, $25-48$.

Elliott, P., Giester, G., Rowe, R., Pring, A., 2014. Putnisite, $\mathrm{SrCaCr}_{8}^{3+}$. $\left(\mathrm{CO}_{3}\right)_{8} \mathrm{SO}_{4}(\mathrm{OH})_{16} \cdot 25 \mathrm{H}_{2} \mathrm{O}$, a new mineral from Western Australia: description and crystal structure. Mineral. Mag. 78, 131-144.

Hughes, J.M., Ni, Y., 1994. A high-precission crystal structure refinement of daqingshanite-(Ce) from Nkombwa Hill carbonatite, Zambia. Mineral. Mag. 58, 493-496.

Kampf, A.R., Rossman, G.R., Steele, I.M., Pluth, J.J., Dunning, G.E., Walstrom, R.E., 2010. Devitoite, a new heterophyllosilicate mineral with astrophyllite-like layers from Eastern Fresno County, California. Can. Mineral. 48, 29-40. 
Kampf, A.R., Mills, S.J., Housley, R.M., Rossman, G.R., Nash, B.P., Dini, M., Jenkins, R.A., 2013. Joteite, $\mathrm{Ca}_{2} \mathrm{CuAl}\left[\mathrm{AsO}_{4}\right]\left[\mathrm{AsO}_{3}(\mathrm{OH})\right]_{2}(\mathrm{OH})_{2}$. $5 \mathrm{H}_{2} \mathrm{O}$, a new arsenate with a sheet structure and unconnected acid arsenate groups. Mineral. Mag. 77, 2811-2823.

Kampf, A.R., Mills, S.J., Housley, R.M., Marty, J., 2014. Lead-tellurium oxysalts from Otto Mountain near Baker, California: IX. Agaite, $\mathrm{Pb}_{3} \mathrm{Cu}^{2+}$. $\mathrm{Te}^{6+} \mathrm{O}_{5}(\mathrm{OH})_{2}\left(\mathrm{CO}_{3}\right)$, a new mineral with $\mathrm{CuO}_{5}-\mathrm{TeO}_{6}$ polyhedral sheets. Am. Mineral. 98, 512-517.

Krause, W., Effenberger, H., Bernhardt, H.J., Medenbach, O., 2008. Skorpionite, $\mathrm{Ca}_{3} \mathrm{Zn}_{2}\left(\mathrm{PO}_{4}\right)_{2}\left(\mathrm{CO}_{3}\right)(\mathrm{OH})_{2} \cdot\left(\mathrm{H}_{2} \mathrm{O}\right)$, a new mineral from Namibia: description and crystal structure. Eur. J. Mineral. 20, 271-280.

Krivovichev, S.V., 2013. Structural complexity of minerals: information storage and processing in the mineral world. Mineral. Mag. 77, 275-326.

Krivovichev, S.V. 2014. Which inorganic structures are the most complex? Angew. Chem. Int. Ed. 53, 654-661.

Krivovichev, S.V., Plásil, J., 2013. Mineralogy and crystallography of uranium, in: Burns, P.C., Sigmon, G.E. (Eds), Uranium: Cradle to Grave. Mineralogical Association of Canada Short Course Ser. Vol. 43, pp. 15120

Krivovichev, S.V., Britvin, S.N., Burns, P.C., Yakovenchuk, V.N., 2002. Crystal structure of rimkorolgite, $\mathrm{Ba}\left[\mathrm{Mg}_{5}\left(\mathrm{H}_{2} \mathrm{O}\right)_{7}\left(\mathrm{PO}_{4}\right)_{4}\right]\left(\mathrm{H}_{2} \mathrm{O}\right)$, and its comparison with bakhchisaraitsevite. Eur. J. Mineral. 14, 397-402.

Krivovichev, S.V., Chernyatieva, A.P., Britvin, S.N., Yakovenchuk, V.N., Krivovichev, V.G., 2013. Refinement of the crystal structure of bonshtedtite, $\mathrm{Na} 3 \mathrm{Fe}\left(\mathrm{PO}_{4}\right)\left(\mathrm{CO}_{3}\right)$. Geol. Ore Deposits 55, 669-675.

Kurova, T.A., Shumyatskaya, N.G., Voronkov, A.A., Pyatenko, Yu.A. (1980) Crystal structure of sidorenkite $\mathrm{Na}_{3} \mathrm{Mn}\left(\mathrm{PO}_{4}\right)\left(\mathrm{CO}_{3}\right)$. Dokl. Akad. Nauk SSSR 251, 605-607.

Lazic, B., Armbruster, T., Savelyeva, V.B., Zadov, A.E., Pertsev, N.N., and Dzierzanowski, P., 2011. Galuskinite, $\mathrm{Ca}_{7}\left(\mathrm{SiO}_{4}\right)_{3}\left(\mathrm{CO}_{3}\right)$, a new skarn mineral from the Birkhin gabbro massif, Eastern Siberia, Russia. Mineral. Mag. 75, 2631-2648.

Liferovich, R.P., Pakhomovsky, Ya.A., Yakubovich, O.V., Massa, W., Laajoki, K., Gehör, S., Bogdanova, A.N., Sorokhtina, N.V., 2000. Bakhchisaraitsevite, $\mathrm{Na}_{2} \mathrm{Mg}_{5}\left[\mathrm{PO}_{4}\right]_{4} \cdot 7 \mathrm{H}_{2} \mathrm{O}$, a new mineral from hydrothermal assemblages related to phoscorite-carbonatite complex of the Kovdor massif, Russia. N. Jb. Miner. Mh. 2000, 402-418.

McDonald, A.M., Chao, G.-Y., Grice, J.D., 1994. Abenakiite-(Ce), a new silicophosphate carbonate mineral from Mont Saint-Hilaire, Quebec: description and structure determination. Can. Mineral. 32, 843-854.

McDonald, A.M., Back, M.E., Gault, R.A., Horváth, L., 2013. Peatite-(Y) and ramikite-(Y), two new $\mathrm{Na}-\mathrm{Li}-\mathrm{Y} \pm \mathrm{Zr}$ phosphate-carbonate minerals from the Poudrette pegmatite, Mont Saint-Hilaire, Quebec. Can. Mineral. 51, 569-596.

Mills, S.J., Christy, A.G., Genin, J.-M.R., Kameda, T., Colombo, F., 2012a. Nomenclature of the hydrotalcite supergroup: natural layered double hydroxides. Mineral. Mag. 76, 1289-1336.
Mills, S.J., Kartashov, P.M., Kampf, A.R., Konev, A.A., Koneva, A.A., Raudsepp, M., 2012b. Cordylite-(La), a new mineral species in fenite from the Biraya Fe-REE deposit, Irkutsk, Russia. Can. Mineral. 50, 1281-1290.

Miyawaki, R., Yokoyama, K. and Husdal, T.A., 2013. Bastnäsite-(Nd), a new Nd-dominant member of the bastnäsite group from the Stetind pegmatite, Tysfjord, Nordland, Norway. Eur. J. Miner. 25, 187-191.

Pekov, I.V., Chukanov, N.V., Britvin, S.N., Kabalov, Yu.K., Göttlicher, J., Yapaskurt, V.O., Zadov, A.E., Krivovichev, S.V., Schüller, W., Ternes, B., 2012. The sulfite anion in ettringite-group minerals: a new mineral species hielscherite, $\mathrm{Ca}_{3} \mathrm{Si}(\mathrm{OH})_{6}\left(\mathrm{SO}_{4}\right)\left(\mathrm{SO}_{3}\right) \cdot 11 \mathrm{H}_{2} \mathrm{O}$, and the thaumasitehielscherite solid-solution series. Mineral. Mag. 76, 1133-1152.

Romming, C., Raade, G., 1986. The crystal structure of heneuite, $\mathrm{CaMg}_{5}$. $\left(\mathrm{CO}_{3}\right)\left(\mathrm{PO}_{4}\right)_{3}(\mathrm{OH})$. N. Jb. Miner. Mh. 1986, 351-359.

Shatskiy, A., Sharygin, I.S., Litasov, K.D., Borzdov, Y.M., Palyanov, Y.N., Ohtani, E., 2013a. New experimental data on phase relations for the system $\mathrm{Na}_{2} \mathrm{CO}_{3}-\mathrm{CaCO}_{3}$ at $6 \mathrm{GPa}$ and $900-1400{ }^{\circ} \mathrm{C}$. Am. Mineral. 98, 2164-2171.

Shatskiy, A., Gavryushkin, P.N., Sharygin, I.S., Litasov, K.D., Kupriyanov, I.N., Higo, Y., Borzdov, Yu.M., Funakoshi, K., Palyanov, Yu.N., Ohtani, E., 2013b. Melting and subsolidus phase relations in the system $\mathrm{Na}_{2} \mathrm{CO}_{3}-\mathrm{MgCO}_{3} \cdot \mathrm{H}_{2} \mathrm{O}$ at $6 \mathrm{GPa}$ and the stability of $\mathrm{Na}_{2} \mathrm{Mg}\left(\mathrm{CO}_{3}\right)_{2}$ in the upper mantle. Am. Mineral. 98, 2172-2182.

Shatskiy, A., Sharygin, I.S., Gavryushkin, P.N., Litasov, K.D., Borzdov, Yu.M., Shcherbakova, A.V., Higo, Y., Funakoshi, K., Palyanov, Yu.N., Ohtani, E., 2013c. The system $\mathrm{K}_{2} \mathrm{CO}_{3}-\mathrm{MgCO}_{3}$ at $6 \mathrm{GPa}$ and 900-1450 ${ }^{\circ} \mathrm{C}$. Am. Mineral. 98, 1593-1603.

Shatskiy, A., Borzdov, Yu.M., Litasov, K.D., Kupriyanov, I.N., Ohtani, E., Palyanov, Yu.N., 2014. Phase relations in the system $\mathrm{FeCO}_{3}-\mathrm{CaCO}_{3}$ at $6 \mathrm{GPa}$ and $900-1700{ }^{\circ} \mathrm{C}$ and its relation to the system $\mathrm{CaCO}_{3}-\mathrm{FeCO}_{3}-$ $\mathrm{MgCO}_{3}$. Am. Mineral. 99, 773-785.

Sheldrick, G.M., 2008. A short history of SHELX. Acta Crystallogr. A64, $112-122$.

Sokolova, E.V., Khomyakov, A.P., 1992. Crystal structure of a new mineral $\mathrm{Na}_{3} \mathrm{SrPO}_{4} \mathrm{CO}_{3}$ from bredleyite group. Dokl. Akad. Nauk SSSR 322, 531-535.

Sokolova, E.V., Yegorov-Tismenko, Y.K., 1990. Crystal structure of girvasite. Dokl. AN SSSR 331, 1372-1376 (in Russian).

Szymanski, J.T., Roberts, A.C., 1990. The crystal structure of voggite, a new hydrated $\mathrm{Na}-\mathrm{Zr}$ hydroxide-phosphate-carbonate mineral. Mineral. Mag. 54, 495-500.

Yakubovich, O.V., Massa, W., Liferovich, R.P., Pakhomovsky, Y.A., 2000. The crystal structure of bakhchisaraitsevite, $\left[\mathrm{Na}_{2}\left(\mathrm{H}_{2} \mathrm{O}\right)_{2}\right]\left\{\left(\mathrm{Mg}_{4.5} \mathrm{Fe}_{0.5}\right)\right.$. $\left.\left(\mathrm{PO}_{4}\right)_{4}\left(\mathrm{H}_{2} \mathrm{O}\right)_{5}\right\}$, a new mineral species of hydrothermal origin from the Kovdor phoscorite-carbonatite complex, Russia. Can. Mineral. 38, 831838. 